www.nature.com/pj

\title{
Phase separation in mixtures of flexible and semiflexible polymers
}

\author{
Narayan P Adhikari and Ekkehard Straube \\ Using off-lattice Monte Carlo simulations, the critical value of the Flory-Huggins parameter, $\chi$, for flexible-semiflexible \\ (isotropic-isotropic) polymer systems as a function of the stiffness of the semiflexible components was estimated. The \\ simulation data were compared with those of the mean field, and it was found that both agree very well. The interfacial \\ tension and the width of the flexible-semiflexible polymer systems were also studied for strong and weak segregation limits. \\ Polymer Journal (2011) 43, 751-756; doi:10.1038/pj.2011.29; published online 20 July 2011
}

Keywords: isotropic-isotropic polymer; phase separation; semiflexible polymers; stiffness disparity

\section{INTRODUCTION}

Different kinds of polymers can be mixed into a single material in different ways, which can lead to a wide range of phase behaviors that directly influence the associated physical properties and applications of polymers. Two different polymers generally do not mix well. The factors that control polymer-polymer phase behavior are the choice of monomers, molecular architecture, composition, molecular size, interaction energies, specific interactions and the 'Equation of state effects'. The study of the structure, phase behavior and interfacial properties of polymers has found longstanding and widespread interest. ${ }^{1-10}$ Understanding the phase behavior of mixtures of different kinds of polymers is important scientifically ${ }^{11}$ as well as practically. The scientific importance arises from the complex behavior polymer mixtures display, and the practical importance arises from the many industrial applications of these materials. Polymer blends are generally structurally asymmetric, corresponding to species-dependent local intramolecular properties, such as monomer shape, branch content, and persistence length. Such asymmetries are expected to have a major impact on blend thermodynamics and phase diagrams and can give rise to non-Flory-Huggins miscibility behavior. The prediction of the phase behavior of semiflexible polymeric materials is an important step toward the full characterization of the structural and dynamic properties of liquid-crystalline polymeric materials. In polymer blends, it is important to know how the locations of various phases, isotropic and nematic, and their transitions depend on the properties of the two components, their rigidities, polymerization indices, interactions and so on.

Differences in chemical structure may also lead to different spatial configurations of the chemical repeat units, corresponding to different persistence lengths, that is, stiffness disparities. ${ }^{4}$ Such stiffness disparities may occur even in materials that are chemically very similar, for example, different polyolefins. Liu and Fredrickson ${ }^{12}$ have calculated a free energy functional for a flexible-semiflexible polymer system that depends on two order parameters, namely, the concentration and orientational density of polymer segments. Usually, the phase behavior of flexible polymers is described in terms of a single order parameter, the concentration. However, owing to the fact that individual monomers may have a rigid, anisotropic character, a description of stiff polymers should include the orientational density as a second order parameter. The model by Holyst and Schick ${ }^{13}$ extracts general relationships between the properties of phase diagrams and polymer parameters. Sheng et al. ${ }^{14}$ have studied the effect of chain stiffness on polymer phase behavior.

In the case of flexible and semiflexible polymer blends, one wants to know how the location of various phases, isotropic and nematic, and their transitions depend on the properties of the two components, their rigidities, polymerization indices, interactions and so on. ${ }^{4}$ The prediction of the phase behavior of semiflexible polymeric materials is an important step toward the full characterization of the structural and dynamic properties of liquid-crystalline polymeric materials. High-performance materials containing blends of small-molecule liquid crystals or liquid-crystalline polymers with flexible polymers are typically multidomain composites. Liquid crystals and liquidcrystalline polymers are quite stiff and consequently mix poorly with flexible polymers. ${ }^{12,15,16}$

Polymer systems with flexible-semiflexible chains have attracted a great deal of attention because different polymers have different stiffnesses. As the stiffness disparity increases, the study of phase separation becomes more difficult. ${ }^{4}$ Usually, the study of phase separation by computer simulation is performed using a semi-grand canonical ensemble in which the long wavelength degrees of freedom are relaxed by the exchange between different types of chains. ${ }^{17}$ When the stiffness disparity increases, such a semi-grand canonical ensemble becomes inefficient as there are many more allowed configurations of 
flexible chains than semiflexible chains. ${ }^{15}$ Therefore, the study of phase transitions in flexible-semiflexible polymer systems using computer simulation is challenging.

The simplest polymer mixture is a blend of two different types of homopolymers. A very fruitful approach to study this type of system near its consolute (critical) point is based on the mean-field (MF) Flory-Huggins free energy and the de Gennes random phase approximation for the scattering intensity. ${ }^{18}$ Both can be stated in terms of the Landau-Ginzburg model. De Gennes pointed out that the MF theory is rather good for high molecular mass mixtures in contrast to low molecular mass mixtures, for which the MF theory breaks down close to the critical point. In fact, in the limit of $N \rightarrow \infty(N$, number of monomers per chain), the MF theory is exact. In other words, because of the chain connectivity, the effective range of interactions between polymers, which is roughly the extension of the chains, becomes very large for high molecular weights, and according to the Ginzburg criterion, the critical region in which critical fluctuations become important is very small as a result. The MF approach is quantitatively correct if the correlation length $\xi \sim\left(T / T_{C}-1\right)$ is comparable or smaller than the typical length scale in the system, that is, the radius of gyration $\sim \sqrt{ } N$. Comparing two length scales for a large $N$, we find that the MF theory breaks down very close to the critical point, that is, for $\left|T-T_{\mathrm{C}}\right| / T_{\mathrm{C}} \sim 1 / N$. This is the Ginzburg criterion.

In the present work, the critical value of the Flory-Huggins parameter was estimated as a function of the stiffness of the semiflexible components in flexible-semiflexible polymer systems. In the next section, the model and techniques are described; in the following section, the results are presented and discussed. We close with a brief discussion of our findings and an outlook on future work.

\section{MODEL AND SIMULATION TECHNIQUES}

We performed computer simulations of mixtures of flexible and semiflexible polymers to estimate the critical value of the Flory-Huggins parameter, $\chi$. Figure 1 shows a typical snapshot of such a system. A coarse-grained continuous-space model has been used, which is described in detail elsewhere. ${ }^{15}$ This off-lattice model has the advantage of providing a more direct way (by setting a restriction on bond angles) of generating semiflexible chains and allows for the investigation of chains with larger stiffness ranges than those under the often used lattice model. ${ }^{17}$ The polymer chains are modeled, using the rod-bead model, as a succession of jointed spherical monomers. Each chain consists of $N$ (with $N=32$ ) spheres of equal diameter $d_{\min }=\sqrt{ } 3$ that are connected by $(N-1)$ bonds of variable length $d_{\min } \leqslant d_{\max } \leqslant(4 / 3) d_{\min }$. A bending restriction has been imposed by a stepwise potential. We have generated semiflexible chains in which the angle between two consecutive bond vectors is not larger than $90^{\circ}, 75^{\circ}$ and $60^{\circ}$, which correspond to semiflexible chains

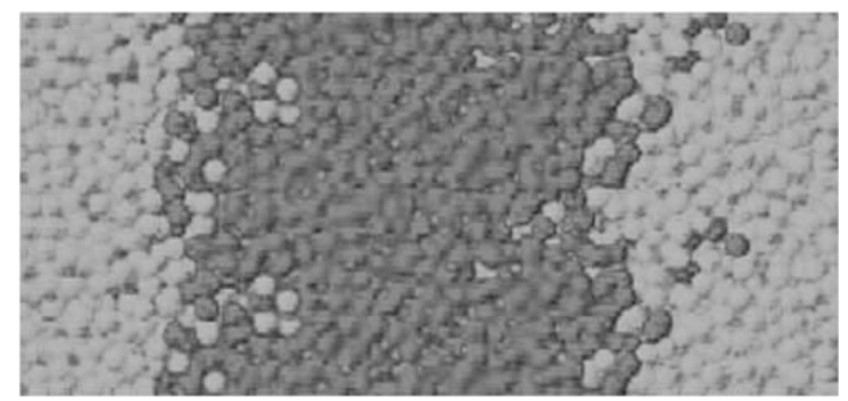

Figure 1 Typical snapshot of systems studied in the present work. Green polymers are flexible, whereas red polymers are semiflexible. The stiffness of the red chains varies. A full color version of this figure is available at Polymer Journal online. with persistence lengths in units of average bond length $\left(l_{\mathrm{p}} / a\right)$ equal to $2.0,2.5$ and 4.0, respectively, where $a$ is the average bond length. In the present model, the flexible chains assume $\left(l_{\mathrm{p}} / a\right)$ values equal to 1.25 . The whole system consists of 512 flexible and semiflexible chains. All chains have an average bond length $a \sim 2$.

The interaction between any indirectly jointed segments is also modeled by a stepwise potential. It is assumed that the interaction between similar monomers $\mathrm{A}$ and $\mathrm{B}$ (type A for flexible chains and type B for semiflexible chains), is symmetric, that is, $V_{\mathrm{AA}}=V_{\mathrm{BB}}=0$, and a repulsive potential with the interaction parameter $\varepsilon$ acts between different monomers $V_{\mathrm{AB}}=k_{\mathrm{B}} T \varepsilon$, where $k_{\mathrm{B}}$ is the Boltzmann constant and $T$ is the absolute temperature. The assumed range of the interaction between two different types of monomers is $d_{\text {int }}=(\sqrt{ } 5 / 3) d_{\min }$. The Flory-Huggins parameter depends on the monomer interaction parameter $\varepsilon$ and also on the average number of interchain contacts for a monomer within a sphere corresponding to the interaction range between two different types of monomers, $z_{\text {eff }}$.

$$
\chi=z_{\text {eff }} \varepsilon
$$

where

$$
Z_{\text {eff }}=\frac{Z_{\text {eff,flexible }}}{2}+\frac{Z_{\text {eff,semiflexible }}}{2} .
$$

Hence, $\chi$ increases slightly with the increasing stiffness of the semiflexible component because in the semiflexible chains the number of contacts between monomers from other chains increases. We generated 32 flexible random walk chains with random bond-length distributions $d_{\min } \leqslant d \leqslant d_{\max }$ with no overlap between the next nearest neighbors within the chain. ${ }^{15}$ Another 32 random walk chains (semiflexible) were generated by setting additional constraints on the angles between two consecutive bond vectors of a chain. We considered an initial box with the dimensions $64 \times 16 \times 16$ as having three compartments. One half of the box was randomly occupied by flexible chains (a quarter at the top and a quarter at the bottom) and the remaining half of the box (the middle) by the semiflexible chains. The overlaps between the segments were then removed by a stepwise increase in the diameter of the spherical monomers followed by Monte Carlo steps. This process was repeated until the minimum distance between any two monomers was equal or greater than $d_{\min }$. After the overlaps were removed, the size of the system was doubled by shifting the $y$ and $z$ coordinates to obtain a system of 256 chains in a $64 \times 32 \times 32$ parallelepiped. Furthermore, we multiplied the system by shifting the $y$ and $z$ coordinates again to obtain a system of study with 1024 chains in a $64 \times 64 \times 64$ cube.

For equilibration and thermodynamic averaging, we performed Monte Carlo steps according to the standard Metropolis algorithm using a random choice of monomer and cyclic choice of one of the six directions along the coordinate axes. The length of an attempted step was randomly chosen to be between zero and a maximum step length $\sim 0.23 \times d_{\text {min }}$. To accelerate the tests for hard core overlapping and the calculations of the interaction energy after each attempted move, we followed the linked-cell method. ${ }^{19}$ This process leads to two well-defined interfaces in the generated canonical (NVT) ensemble $(L \times L \times L)$, with periodic boundary conditions along all three directions. The interfaces are, on average, located in $1 / 4$ and $3 / 4$ of the $x$ dimensions of the simulation box.

To determine whether we had reached equilibrium configurations, we monitored the square of the interfacial width $\left(w^{2}\right)$ and the mean squared displacement (MSD) of the center of mass of chains against time. A system with interfaces in the weak segregation limit (WSL) may be expected to be in equilibrium if the MSD of the center of mass of chains after the removal of the overlaps is comparable to the square of the interfacial width. In the strong segregation limit (SSL), it is sufficient to monitor the square of the radius of gyration, $R_{\mathrm{g}}^{2}$, rather than $w^{2}$, as in the case when $R_{\mathrm{g}}^{2}>w^{2}$. In WSL, $w^{2}>R_{\mathrm{g}}^{2}$, so we monitored $w^{2}$. Owing to confinement along the perpendicular direction caused by the two interfaces in the system, the perpendicular and parallel components of the MSD (parallel and perpendicular with respect to the plane of the interface, see the Figure 1) are not equal because of chain orientation, ${ }^{15}$ and the MSDs also become increasingly different with increasing displacement. Figure 2 presents the MSD and the square of interfacial width for a system of semiflexible chains and flexible chains in which the semiflexible component has 


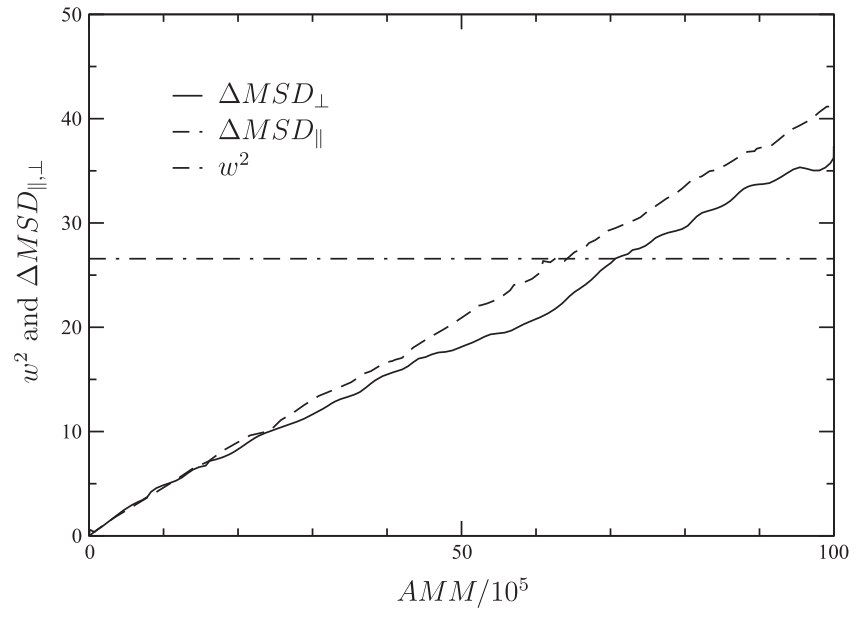

Figure 2 Mean squared displacement (MSD) of the center of mass of polymers and square of the interfacial width versus the attempted number of moves per monomer (AMM) in the weak segregation limit for a flexiblesemiflexible system in which the semiflexible polymers have persistence length $\left(I_{p} / a\right)=4.2$ when $\chi=0.136$.

a persistence length $\left(l_{\mathrm{p}} / a\right)$ equal to 4.2 , and the Flory-Huggins parameter $(\chi)$ of the system is 0.136 . The attempted number of moves per monomer required to reach an equilibrium configuration also varies with $\chi$ and the flexibility of the semiflexible component. For a system with a persistence length of the semiflexible component equal 4.2 and $\chi$ equal to $0.085,10^{8}$ attempted number of moves per monomer were performed to reach a configuration sufficiently close to equilibrium. Measurements of the interfacial tensions were performed after testing the equilibrium of the systems.

In the present work, because of the stiffness disparity between the species, the straightforward application of the semi-grand-canonical identity changes between different polymer types became rather inefficient with increasing stiffness disparity. We determined the interfacial tension with the virial theorem. One can also estimate the interfacial tension by analyzing the spectrum of capillary fluctuations, ${ }^{15}$ but in the present work, we used the virial theorem. Determining the interfacial tension using the virial theorem rests on the determination of the anisotropy of the pressure tensor of a system with an interface. The interfacial tension $\sigma$ can be expressed as follows:

$$
\sigma=\frac{\Delta F}{\Delta A}
$$

where $\Delta F$ is the difference in the free energy on changing the cross-sectional area of the interface by $\Delta A$. The change in the free energy can be calculated by considering the forces caused by a small deformation of the simulation box. This results in the following:

$$
\sigma=\frac{f_{\perp} L_{\perp}}{L_{\|}^{2}}-\frac{f_{\|}}{L_{\|}}
$$

where $f_{\perp, \|}$ are the normal forces acting on the boundary of the simulation box perpendicular and parallel to the interface plane. The force can be measured by a small homogeneous uniaxial deformation of the chains. In the present work (for the step potential, $U_{\mathrm{i}}$ ), the force components were calculated as follows:

$$
\frac{f_{\perp, \|} L_{\perp, \|}}{k_{\mathrm{B}} T}=\frac{1}{\Delta \alpha} \sum_{i}\left\langle\Delta W_{i} M_{i}(\alpha, \alpha \pm \Delta \alpha)\right\rangle
$$

where $i$ is the type of interaction,

$$
\Delta W_{i}=\Delta\left(\exp \left(-U_{i} / k_{\mathrm{B}} T\right)\right)
$$

is the change in weight and

$$
M_{i}(\alpha, \alpha \pm \Delta \alpha)
$$

is the number of monomers entering/leaving the interaction range for $i$ of another monomer when changing the compression/expansion from $\alpha$ to $\alpha \pm \Delta \alpha$.

In the present work, the change in the interfacial tension and width as a function of $\chi$ were studied, covering both the SSL and the WSL. The simulation results were compared with the MF results. In SSL, MF theory predicts that the interfacial tension varies as the square root of the Flory-Huggins parameter, $\chi^{: 20}$

$$
\frac{\sigma}{k_{\mathrm{B}} T}=\frac{2}{3} \sqrt{\alpha}\left(\frac{\left(\beta_{\mathrm{A}}^{3}-\beta_{\mathrm{B}}^{3}\right)}{\left(\beta_{\mathrm{A}}^{2}-\beta_{\mathrm{B}}^{2}\right)}\right)
$$

$\beta \mathrm{i}(\mathrm{i}=A, B)$, such that

$$
\beta_{i}=\sqrt{\left(\frac{1}{6} \rho_{0 i} b_{i}\right)}
$$

are the parameters that contain the chain statistics. The statistical segment lengths $b i$ are defined in the same way as in the reference ${ }^{15}$ and $\rho_{0 i}$ are the number densities of statistical segments in both bulk phases, respectively. The interaction parameter $\alpha$ of the interaction between two statistical segments is then given by

$$
\alpha=\rho_{0} \chi
$$

with the Flory-Huggins parameter $\chi$ for the interaction of two beads of chains of different kinds defined by equation (1).

In the WSL, the Flory-Huggins-de Gennes formula for interfacial tension is ${ }^{5}$

$$
\frac{\sigma}{k_{\mathrm{B}} T}=\frac{9}{b^{2} \sqrt{N}}\left(1-\frac{\chi_{\mathrm{C}}}{\chi}\right)^{3 / 2}
$$

where $b$ is the statistical segment length, $N$ is the number of monomers per chain and $\chi_{C}$ is the critical value of $\chi$. To derive equation (10), it is assumed that the statistical segment length for two types of chains is equal. If one considers an asymmetrical system, the prefactor will be different; however, the exponents will remain the same. From equation $(10),\left(\sigma / k_{\mathrm{B}} T\right)^{2 / 3}$ linearly varies with $(1 / \chi)$, and the interfacial tension vanishes when $\chi=\chi_{\mathrm{C}}$. Thus, by studying the behavior at low values of $\chi$ for the interfacial tension and extrapolating to zero, one can estimate the critical value of $\chi$.

The behavior of the interfacial width at low $\chi$ has also been studied. In the WSL, the interfacial width $w$ is given by

$$
W=\frac{b \sqrt{N}}{3}\left(\frac{\chi}{\chi_{C}}-1\right)^{(-1 / 2)}
$$

In equation (11), $b$ and $N$ carry the same meanings as in equation (10). From equation (11), it is clear that the width $w$ will be infinite at $\chi=\chi_{\mathrm{C}}$. We substitute $N \chi_{C}=2$ in equation (10) and use the same mapping as those in ${ }^{15}$ to compare the simulation data with the MF data.

\section{RESULTS AND DISCUSSION}

Figures 3-6 show the interfacial tension $\left(\sigma / k_{\mathrm{B}} T\right)^{2 / 3}$ as a function of $(1 / \chi)$ for different stiffnesses (persistence length, $\left(l_{\mathrm{p}} / a\right)=1.25,2.00$, 2.50 and 4.20) of the semiflexible components. As described in the previous section (see equation (10)), $\left(\sigma / k_{\mathrm{B}} T\right)^{2 / 3}$ varies linearly with $(1 / \chi)$ in the WSL, and the interfacial tension vanishes when $\chi=\chi_{\mathrm{C}}$. Figure 3 shows the dependence of $\left(\sigma / k_{\mathrm{B}} T\right)^{2 / 3}$ on $(1 / \chi)$ for a flexibleflexible polymer system. We can describe the low $\chi$ behavior of $\left(\sigma / k_{\mathrm{B}} T\right)^{2 / 3}$ as a straight line, as shown in WSL, which enables us to estimate the value of $\chi$ for which $\left(\sigma / k_{\mathrm{B}} T\right)^{2 / 3}$ vanishes. Furthermore, Figure 3 shows the interfacial tension in the SSL and a curve describing its behavior (see equation (7)). It can be seen in the figure that the data for the SSL do not follow the straight line fitted for the 


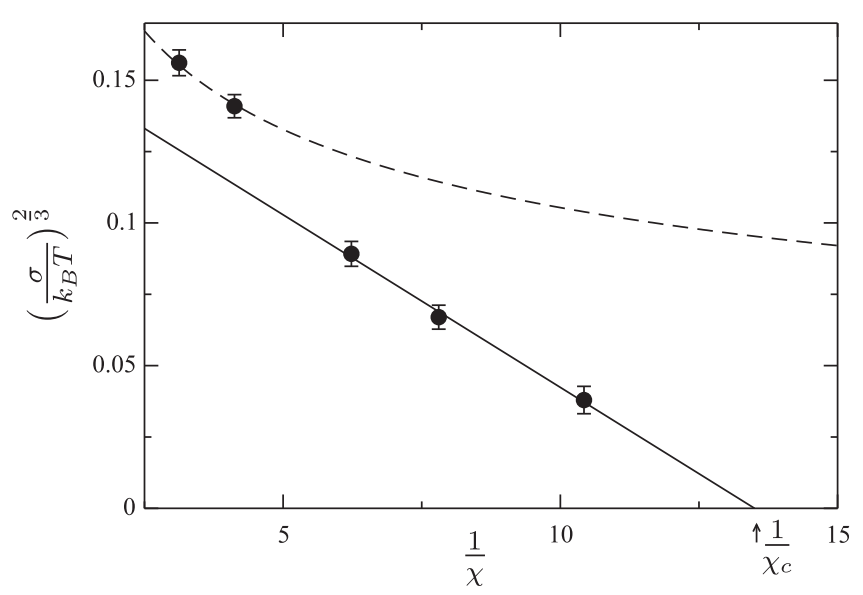

Figure $3\left(\sigma / k_{B} T\right)^{2 / 3}$ versus $(1 / \chi)$ in a flexible-flexible polymer system. The solid line is a fit to the weak segregation data, and the dashed line is the curve of the formula in the strong segregation limit (equation (7)).

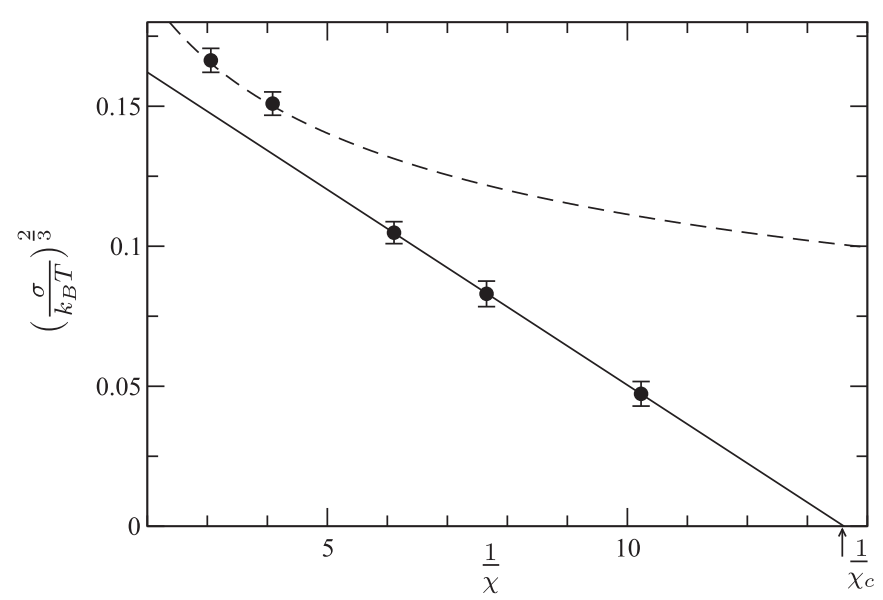

Figure $4\left(\sigma / k_{\mathrm{B}} T\right)^{2 / 3}$ versus $(1 / \chi)$ in a flexible-semiflexible polymer system in which the semiflexible component has persistence length $\left(I_{p} / a=2.0\right)$. The solid line is a fit to the weak segregation data, and the dashed line is the curve of the formula in the strong segregation limit (equation (7)).

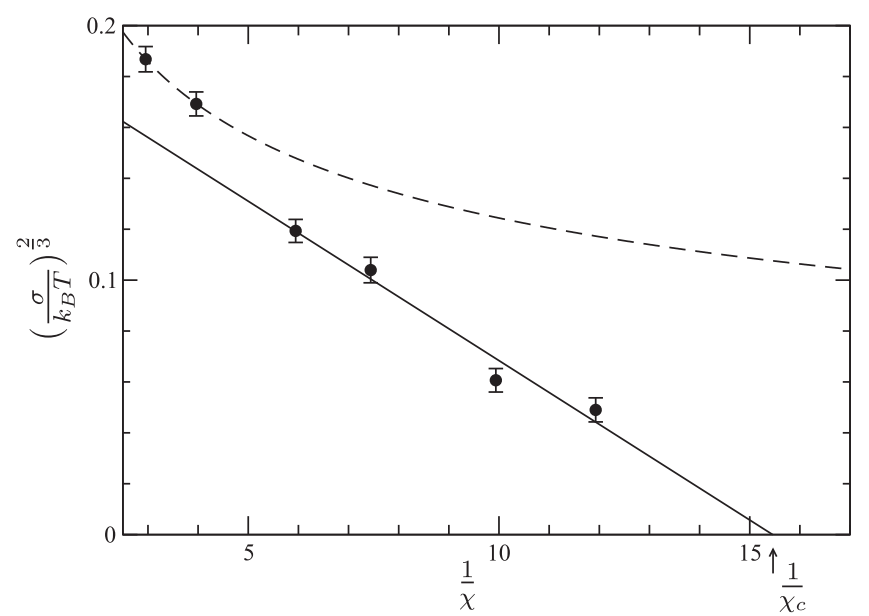

Figure $5\left(\sigma / k_{\mathrm{B}} T\right)^{2 / 3}$ versus $(1 / \chi)$ in a flexible-semiflexible polymer system in which the semiflexible component has persistence length $\left(I_{\mathrm{p}} / a=2.5\right)$. The solid line is a fit to the weak segregation data, and the dashed line is the curve of the formula in the strong segregation limit (equation (7)).

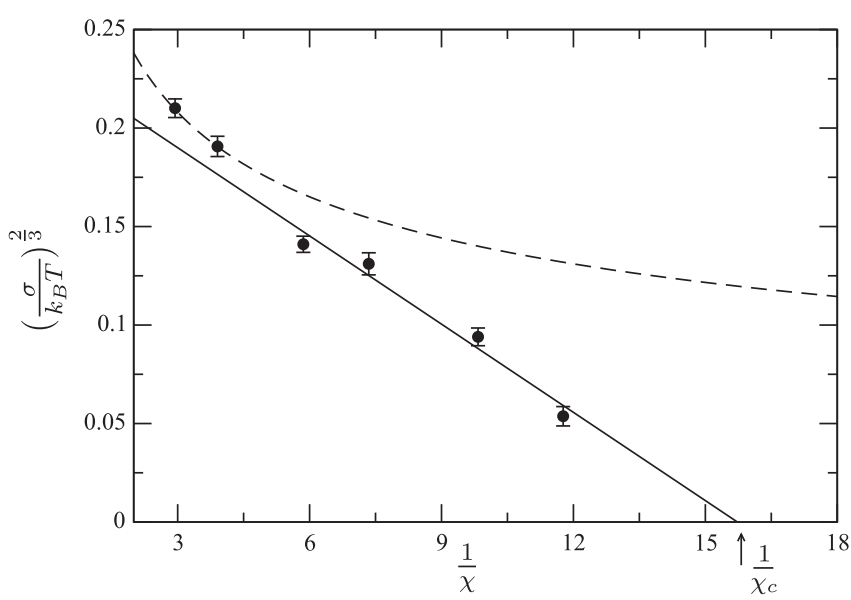

Figure $6\left(\sigma / k_{\mathrm{B}} T\right)^{2 / 3}$ versus $(1 / \chi)$ in a flexible-semiflexible polymer system in which the semiflexible component has persistence length $\left(/ I_{\mathrm{p}} / a=4.2\right)$. The solid line is a fit to the weak segregation data, and the dashed line is the curve of the formula in the strong segregation limit (equation (7)).

Table 1 The prefactors in equations (7) and (10) obtained after comparing our data with the mean field (MF)

Systems (I /a) Equation (7) Equation (10) Equation (7) MF Equation (10) MF

\begin{tabular}{lcccc}
\hline 1.25 & 0.1840 & 0.109 & 0.1744 & 0.125 \\
2.00 & 0.1886 & 0.121 & - & 0.145 \\
2.50 & 0.1939 & 0.140 & - & 0.165 \\
4.20 & 0.2283 & 0.165 & - & 0.192 \\
\hline \multicolumn{4}{l}{ Here, / $/$ 's are in units of the average bond length (a). In the column of system means, the }
\end{tabular}

Here, Ip's are in units of the average bond length (a). In the column of system means, the
system contains flexible polymers and semiflexible polymers of given persistence length.

data in the WSL. From the fitted straight line for the interfacial tension in the WSL, we have estimated the critical value of $\chi$.

The critical value of $\chi$ was also estimated for flexible-semiflexible polymer systems in which the semiflexible components had persistence lengths $\left(l_{\mathrm{p}} / a=\right) 1.25,2,2.5$ and 4.2 . The method used was the same as that described for flexible-flexible polymer systems. The interfacial tensions for these systems are presented in Figures 4-6 for the entire range of $\chi$ that was studied. Furthermore, the prefactors in equation (10) were also calculated. Table 1 shows the prefactors in equation (10), which were determined using the MF and present work. The prefactors from the MF and present work are not very dissimilar. The difference between them is $<5 \%$.

As described above, the critical value of $\chi$ was estimated as a function of the stiffness of the semiflexible components in our systems of study. We studied flexible-flexible and flexible-semiflexible (with varying persistence length $l_{\mathrm{p}} / a$ of semiflexible components up to 4.2, in units of bond length) polymer blends. A comparison of our estimated value of critical $\chi$ with that of Werner et al. ${ }^{21}$ for the flexible-flexible polymer blend shows that our result $\left(\chi_{C}=0.0795\right)$ for this system is not much different from their result $\left(\chi_{C}=0.08\right.$, estimated from their graph).

Figure 7 shows the dependence of the total interfacial width on the Flory-Huggins parameter, $\chi$, for a flexible-semiflexible polymer blend. Figure $7 \mathrm{a}$ shows the dependence of the interfacial width as a function of $\chi$ for a flexible-flexible polymer blend. From Figure $7 \mathrm{a}$, it can be seen that the interfacial width diverges at extremely low values of $\chi$. Werner et al. ${ }^{21}$ have also studied the behavior of interfacial tension, 
a

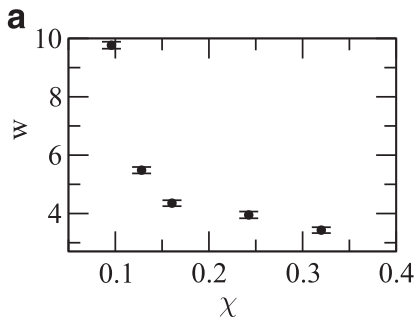

C

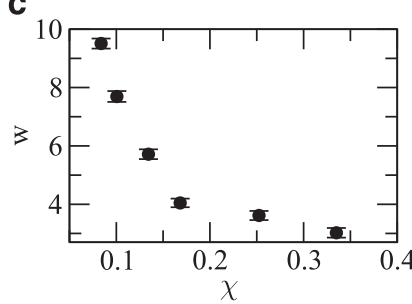

b

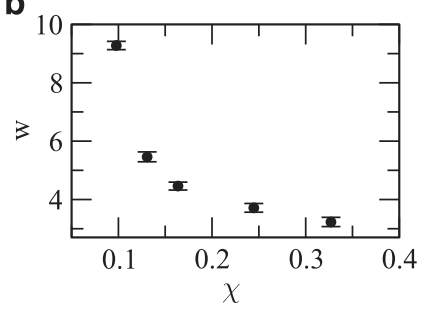

d

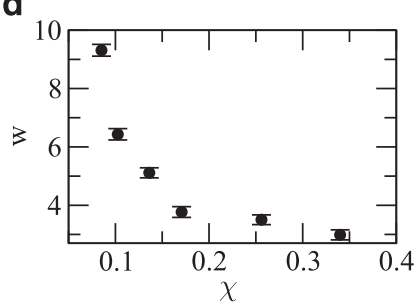

Figure 7 Total interfacial width as a function of the interaction parameter, $\chi$, for a mixture of flexible and semiflexible polymers: (a) flexible-flexible mixture $\left(I_{\mathrm{p}} / a\right)=1.25$, (b) flexible and $(/ / \mathrm{p} / a)=2.0$, (c) flexible and $\left(I_{\mathrm{p}} / a\right)=2.50$ and (d) flexible and $(/ \mathrm{p} / \mathrm{a})=4.20$.

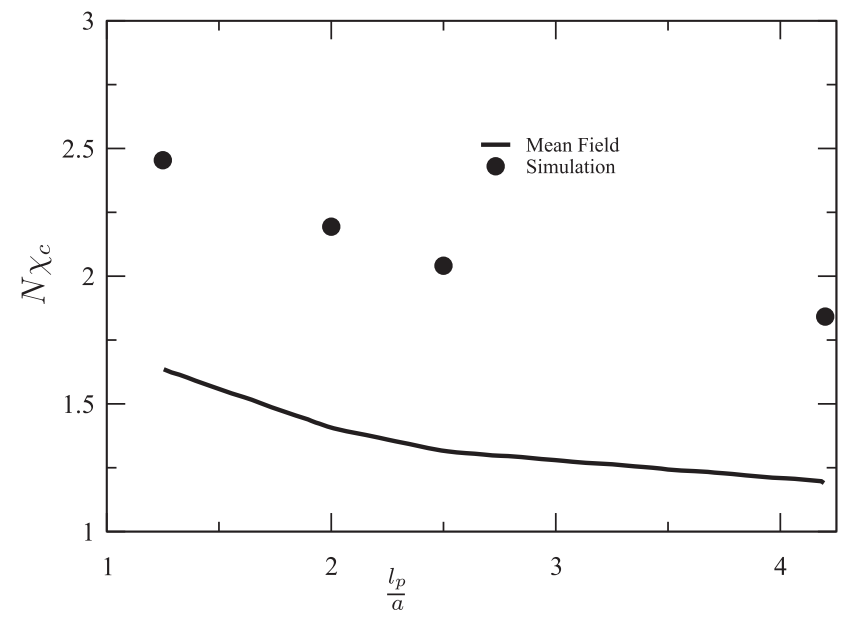

Figure $8 \mathrm{~N} \chi_{c}$ as a function of stiffness of the semiflexible components.

width and other interfacial properties in the WSL using Monte Carlo techniques. Similarly, Figures $7 \mathrm{~b}, \mathrm{c}, \mathrm{d}$ show the interfacial width as a function of $\chi$ for a system of flexible-semiflexible polymers in which the semiflexible chains have persistence lengths $\left(l_{\mathrm{p}} / a\right)=2.0,2.5$ and 4.2, respectively. It can be seen from the figures that the interfacial width increases with the decrease in the interfacial tension. The interfacial width will be infinite at the critical value of $\chi$. The $\chi_{C}$ value determined from our simulations was compared with the MF value for critical $\chi\left(\chi_{\mathrm{C}}\right)$. From the MF theory, one obtains the following: ${ }^{5}$

$$
N \chi_{\mathrm{C}}=\frac{1}{2}\left(\frac{1}{\left(C_{1 N \mathrm{~A}}\right)^{1 / 4}}+\frac{1}{\left(C_{1 N \mathrm{~B}}\right)^{1 / 4}}\right)^{2}
$$

where $C_{1 N i}(i=\mathrm{A}, \mathrm{B})$ is the characteristic ratio of the $i$ th component as defined in (ref. 15) and $N$ is the number of monomers in a chain. The value of $N \chi_{C}$ as a function of the stiffness of the semiflexible

component in a blend of flexible-semiflexible polymers has been calculated using the formula above and from simulation data. Figure 8 shows both types of data. They both show that the value of $N \chi_{C}$ decreases with an increase in the stiffness of the semiflexible polymers. The MF theory gives lower values of $N \chi_{C}$ than the simulation, which is not unexpected, as the MF theory neglects the fluctuations. The nature of both types of data is the same.

\section{CONCLUSIONS AND OUTLOOK}

In the present work, we have estimated the critical value of $\chi$, that is, $\chi_{C}$, as a function of the stiffness of the semiflexible components in flexible and semiflexible polymer blends using off-lattice Monte Carlo simulations for the case of mixing of polymers with different stiffnesses. The semiflexible components had the following persistence lengths $(l \mathrm{p})$, in units of bond length $(a):(l \mathrm{p} / a)=2.0,2.5$ and 4.2 . The flexible component had a persistence length $(l \mathrm{p} / a)=1.25$. The critical values of $\chi$ were estimated for all four systems studied. The estimated critical values of $\chi$ for flexible-flexible polymer systems agree with the previously reported data of Werner et al. ${ }^{21}$ within $<1 \%$. The estimated data for $\chi_{C}$ were compared with those obtained from the MF theory, and it was observed that the data agree at least qualitatively. The MF data estimate values that are lower than those determined in the present work. This may be due to the negligence of fluctuations in the MF theory.

The present work can be extended to study mixtures of rod-like polymers and flexible polymers to consider all of the possible flexibilities of the semiflexible components and of rigid rod-like polymers in the kinds of systems discussed in the present work.

\section{ACKNOWLEDGEMENTS}

NPA acknowledges the financial support of the Graduiertenkolleg 'Polymerwissenschaften' of DFG. Furthermore, support from the Abdus Salam International Center for Theoretical Physics as an Associate Member and study leave from the Central Department of Physics, Tribhuvan University, Kathmandu, Nepal helped to complete this work.

1 Harmandari, V. A., Adhikari, N. P., van der Veg, N. F. A. \& Kremer, K. Hierarchical modeling of polystyrene: from atomistic to coarse-grained simulations. Macromolecules 39, 6708-6719 (2006).

2 Harmandaris, V. A., Adhikari, N. P., van derVeg, N. F. A., Kremer, K., Mann, B. A., Voelkel, R., Weiss, H. \& CheeChin, L. Ethylbenzene diffusion in polystyrene: united atom atomistic/coarse grained simulations and experiments. Macromolecules 40, 7026-7035 (2007).

3 Bates, F. S. Polymer-polymer phase behaviour. Science 251, 898-905 (1991).

4 Adhikari, N. P. Interfacial properties and phase behavior of unsymmetric polymer blends, PhD thesis, Martin-Luther-Univ., Halle-Wittenberg, Germany, (2001).

5 Binder, K. Phase transitions in polymer blends and block copolymer melts: some recent developments. Adv. Polym. Sci. 112, 181-297 (1994).

6 Binder, K. Phase transitions in polymer blends and block copolymer melts in thin films. Ad. Polym. Sci. 138, 1-89 (1999).

7 Mueller, M., Binder, K., Schmid, F. \& Werner, A. Intrinsic profiles and capillary waves at interfaces between coexisting phases in polymer blends. Adv. Colloid Interface Sci. 94, 237-248 (2001).

8 Binder, K. Simulations of phase transitions in macromolecular systems. Comp. Phys. Commun. 147, 22-33 (2002).

9 Holyst, R. \& Vilgis, T. A. The structure and phase transitions in polymer blends, diblock copolymers and liquid crystalline polymers: The Landau-Ginzburg approach. Macromol. Theory Simul. 5, 573-643 (1996).

10 Mueller, M. \& Schmid, F. in Annual Reviews of Computational Physics VI (ed. Stauffer, D.) pages 59-127 (World Scientific, Singapore, 1999).

11 Mueller, M. \& Werner, A. Interfaces between highly incompatible polymers of different stiffness: Monte carlo simulations and self-consistent field calculations. J. Chem. Phys. 107 (1997).

12 Liu, A. J. \& Fredrickson, G. H. Free energy functionals for semiflexible polymer solutions and blends. Macromolecules 26, 2817 (1993).

13 Holyst, R. \& Schick, M. Copolymers as amphiphiles in ternary mixtures: an analysis employing disorder, equimaxima, and lifshitz lines. J. Chem. Phys. 96, 7728 (1992). 
14 Sheng, Y. J., Panagiotopoulos, A. Z. \& Kumar, S. K. Effect of chain stiffness on polymer phase behavior. Macromolecules 29, 4444-4446 (1996).

15 Adhikari, N. P., Auhl, R. \& Straube, E. Interfacial properties of flexible and semiflexible polymers. Macromol. Theory Simul. 11, 315-325 (2002).

16 Adhikari, N. P. \& Straube, E. Interfacial properties of asymmetric polymer mixtures. Macromol. Theory Simul. 12, 499-507 (2003).

17 Binder, K. (ed.) Monte Carlo and Molecular Dynamics Simulations in Polymer Science (Oxford University Press, Oxford, 1995).
18 de Gennes, P. G. Scaling Concepts in Polymer Physics (Cornell University Press, Ithaca and London, 1979).

19 Allen, M. P. \& Tildesley, D. J. Computer Simulations of Liquids (Oxford University Press, Oxford, 1991).

20 Helfand, E. \& Sapse, A. M. Theory of unsymmetric polymerpolymer interfaces. J. Chem. Phys. 62, 1327-1331 (1975).

21 Werner, A., Schmid, F., Mueller, M. \& Binder, K. Intrinsic profiles and capillary waves at homopolymer interfaces: a Monte Carlo study. Phys. Rev. E 59, 728-738 (1999). 